\title{
Neural ECM mimetics
}

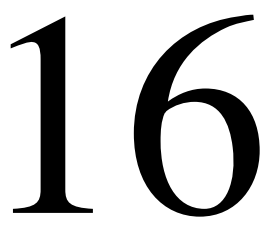

\author{
Veronica Estrada*, Ayse Tekinay , Hans Werner Müller*,1 \\ *Molecular Neurobiology Laboratory, Department of Neurology, Heinrich-Heine-University \\ Medical Center Düsseldorf, Düsseldorf, Germany \\ ¿UNAM-National Nanotechnology Research Center, Institute of Materials Science and \\ Nanotechnology, Bilkent University, Ankara, Turkey \\ ${ }^{1}$ Corresponding author: Tel.: +49-(0)211-811-8410; Fax: +49-(0)211-811-8411, \\ e-mail address: hanswerner.mueller@uni-duesseldorf.de
}

\section{Abstract}

The consequence of numerous neurological disorders is the significant loss of neural cells, which further results in multilevel dysfunction or severe functional deficits. The extracellular matrix $(E C M)$ is of tremendous importance for neural regeneration mediating ambivalent functions: ECM serves as a growth-promoting substrate for neurons but, on the other hand, is a major constituent of the inhibitory scar, which results from traumatic injuries of the central nervous system. Therefore, cell and tissue replacement strategies on the basis of ECM mimetics are very promising therapeutic interventions. Numerous synthetic and natural materials have proven effective both in vitro and in vivo. The closer a material's physicochemical and molecular properties are to the original extracellular matrix, the more promising its effectiveness may be. Relevant factors that need to be taken into account when designing such materials for neural repair relate to receptor-mediated cell-matrix interactions, which are dependent on chemical and mechanical sensing. This chapter outlines important characteristics of natural and synthetic ECM materials (scaffolds) and provides an overview of recent advances in design and application of ECM materials for neural regeneration, both in therapeutic applications and in basic biological research.

\section{Keywords}

Extracellular matrix, Neurodegeneration, Neuronal regeneration, ECM mimetic, Scaffold

\section{ECM MIMETICS ON THE RISE}

The loss of nerve cells is a general and major symptom of many disorders of the nervous system. After a traumatic injury, the differences in the regenerative response of affected neurons of the peripheral nervous system (PNS) versus the central nervous 
system (CNS) become obvious: While neurons of the PNS reveal a significant degree of regeneration after injury, such regenerative neuronal growth generally fails after an injury of the adult CNS although central neurons have some, but very limited, inherent regenerative capacity (David and Aguayo, 1981). The regenerative failure after CNS trauma and neurodegeneration, therefore, cannot be attributed to just one single cause, and the reasons for the difference in the regenerative responses of PNS and CNS are manifold (Ferguson and Son, 2011) including (i) the presence of myelin-associated inhibitors, (ii) a slower rate of degeneration of the distal segment of the injured fiber tract, (iii) a generally slower axonal growth rate, and (iv) the inhibitory influences of the glial and the extracellular environment. Neurodegeneration does, however, not only occur after CNS trauma. Neurodegenerative disorders like Alzheimer's disease, Parkinson's disease, stroke, multiple sclerosis, and amyotrophic lateral sclerosis result in the shared symptom of neuroinflammation, which eventually leads to the dysfunction or even the loss of neurons (Benowitz and Popovich, 2011; Cartier et al., 2005). Because of a general significant increase of the aging population and the consequence of an increased incidence of neurodegenerative diseases, the development of therapies, which may help to rescue and replace the affected cells and thereby preserve the associated functions, is of highest priority. The ECM is, therefore, an important target for the development of therapies for disorders of the CNS, which involve progressive neurodegeneration. Such interventions either directly target the inhibitory environment and its associated molecules, or they function on the basis of replacement strategies. For the latter, ECM mimetics (natural or synthetic matrix materials that mimic the characteristics of native ECMs) provide promising means for cell- or matrix-based therapeutic treatments.

The previous chapters have described the neural ECM and its role in a variety of healthy normal and injury-related processes in full detail. Chapters 16 and 17 discuss current therapies that directly target the neural ECM. However, if such respective therapies cannot be applied or are not efficient, e.g., in case of large tissue defects, or if in vivo differentiation is intended after cellular transplantation, it may be necessary to reconstruct the affected tissue. For example, via the application of suitable matrix mimetics, a growth-promoting microenvironment can be created. This chapter focuses on scaffold materials that are currently used as neural ECM mimetics in basic research and experimental therapies (Figs. 1-3).

\section{THE EXTRACELLULAR MATRIX}

A brief outline of the natural ECM will illustrate its complexity and suggest the difficulty and possible obstacles for the design of ECM mimetics: The ECM is composed of a three-dimensional meshwork of fibrillar proteins and glycosaminoglycans (GAGs) providing an interstitial matrix or basement membrane. The interstitial matrix consists of polysaccharide gels and fibrillar proteins, whereas sheetlike depositions of ECM are characteristic for basement membranes. The molecular ECM components are GAG-containing proteoglycans (chondroitin sulfate, heparan sulfate, and keratin sulfate), nonproteoglycans containing polysaccharide hyaluronic 


\begin{tabular}{|c|c|c|}
\hline $\begin{array}{l}\text { Application of } \\
\text { neural ECM } \\
\text { mimetics }\end{array}$ & Cell-based & A-cellular \\
\hline $\begin{array}{l}\text { Biomedical } \\
\text { research }\end{array}$ & $\begin{array}{l}\text { Cell/tissue } \\
\text { replacement, cell } \\
\text { implantation, } \\
\text { bioengineering }\end{array}$ & \\
\hline $\begin{array}{l}\text { Biomedical } \\
\text { research }\end{array}$ & $\begin{array}{l}\text { Bridging of tissue } \\
\text { defects }\end{array}$ & $\begin{array}{l}\text { Bridging of tissue } \\
\text { defects }\end{array}$ \\
\hline $\begin{array}{l}\text { Biomedical } \\
\text { research }\end{array}$ & Oxidative stress & \\
\hline $\begin{array}{l}\text { Biomedical } \\
\text { research }\end{array}$ & $\begin{array}{l}\text { Growth factor } \\
\text { delivery }\end{array}$ & $\begin{array}{l}\text { Growth factor } \\
\text { delivery }\end{array}$ \\
\hline Medical application & $\begin{array}{l}\text { Minimization of } \\
\text { secondary injury } \\
\text { events }\end{array}$ & $\begin{array}{l}\text { Minimization of } \\
\text { secondary injury } \\
\text { events }\end{array}$ \\
\hline Medical application & PNS/CNS trauma & PNS/CNS trauma \\
\hline Medical application & $\begin{array}{l}\text { Neurodegenerative } \\
\text { conditions }\end{array}$ & \\
\hline Medical application & Neuroinflammation & Neuroinflammation \\
\hline
\end{tabular}

\section{FIGURE 1}

Possible applications of neural ECM mimetics. Cell-based and a-cellular scaffolds are suitable matrices for numerous strategies in both basic biomedical research and medical therapy.

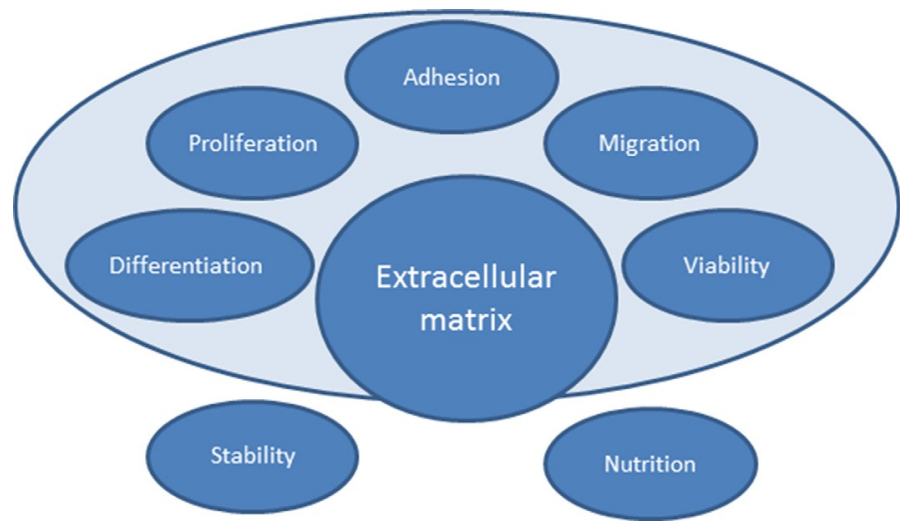

\section{FIGURE 2}

Basic functions of the natural extracellular matrix. One important general feature, which is exerted by the ECM, is the provision of a physically stable scaffold. The maintenance of ion homeostasis and the exchange of nutrients are vital for the correct function of cellular networks and tissues. ECM characteristics, which are especially important for cell-cell and cell-matrix interactions, respectively, and which are mediated by a delicate ECM meshwork, are highlighted by the light blue ellipse. These functions comprise the cellular processes of differentiation, migration, adhesion, proliferation, and viability. 


\section{CHAPTER 16 Neural ECM mimetics}
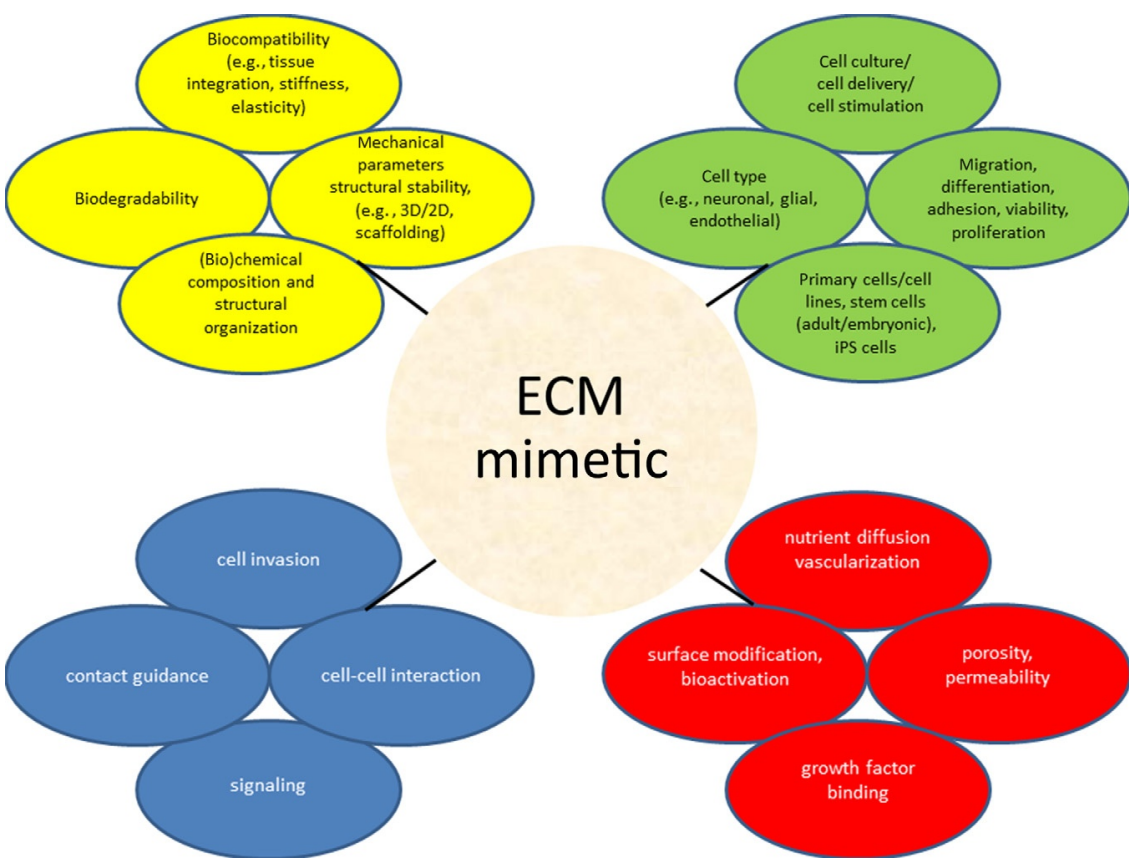

\section{FIGURE 3}

Requirements and considerations for the design of ideal neural extracellular matrix mimetics. The more closely an ECM mimetic resembles the natural ECM, the better it can exert the necessary functions. In addition to mechanical parameters, which strongly influence the cellular behavior and tissue stability, general features (yellow (light gray in print version)) of an ECM mimetic include its biocompatibility, biodegradability, and its general composition. Many characteristics, which are cell-specific (green (gray in print version)), would be considered for neural ECM design in cell replacement and general cell implantation strategies, for example, the cell type, whereas other basic functional requirements also apply for the general cellular behavior in a matrix (e.g., migration and differentiation). The different requirements certainly intermingle in an ideal neural ECM mimetic and, therefore, should not be considered independently of each other. For instance, cell-cell interactions are very important cell-related functions, but they are also vital for the structural organization, biocompatibility, etc. and for complex functions (blue (dark gray in print version)) such as signaling or contact guidance. Finally, synthetic and natural ECM mimetics can be designed and "tuned" to allow very precise functions (red (black in print version)). This can be achieved by the addition of bioactive groups to a scaffold material (surface modification) or by incorporating different degrees of porosity. Thereby, vascularization and subsequently nutrient exchange of an extracellular matrix can be influenced.

acid (HA), fibers of collagen or elastin, and connecting proteins such as fibronectin or laminin. Due to their water-attracting features, proteoglycans keep the ECM well hydrated. Furthermore, they aid in the entrapment and the storage of growth factors. Collagens, the most abundant proteins of the ECM, are mostly fibrillary proteins that provide structural and tensile support for cellular bonds. Exceptions are collagens 
of the nonfibrillary type including collagen IV, which forms the sheetlike meshed, nonfibrillar network of the basement membrane. The important functions that are common to ECMs in general are cell adhesion, cell communication, and cell differentiation. ECMs provide structural stability to tissues. The formation of ECM is essential for several vital processes, such as wound healing, growth, and fibrosis. Cell-ECM interactions are highly dynamic bidirectional processes, which require a very complex temporal and spatial coordination of signaling, receptor-mediated transmission, and regulation of gene expression for their correct function. The three main effectors in a native ECM microenvironment are insoluble hydrated macromolecules, soluble macromolecules, and surface proteins of neighboring cells (Lutolf and Hubbell, 2005). The molecular interaction of a cell with these effectors will determine its ultimate fate, i.e., differentiation, proliferation, migration, apoptosis, or other specific functions.

CNS trauma research models (e.g., experimental spinal cord injury) are useful in order to explore the suitability of different materials as possible ECM mimetics in vivo: large tissue defects that result from primary and secondary injury events (e.g., inflammation, scarring, or cavity formation) and the pathological changes in the native ECM require the removal and/or the reconstruction of the affected tissue in order to restore tissue continuity. A variety of scaffold-based strategies exist in the field of experimental CNS trauma, which involve either cellular transplantation or the implantation of matrix materials as bridging substrates or a combination of both. In vitro cell culture experiments often focus on the survival, migration, and differentiation of the cells in a suitable matrix. In vivo cell transplantation approaches often target similar effects, but the complex and mostly inhibitory environment requires additional considerations.

\section{INTERACTION OF ECM SCAFFOLD AND HOST ECM}

The biocompatibility or the interaction of an artificial scaffold material with host tissue can be modulated via the surface characteristics of the material. Different conditions require different treatments and, therefore, different matrix characteristics. While for cell replacement strategies the supply with factors that mediate cellular differentiation, migration, and survival is of high priority, the degree of biodegradability is a very important issue in regenerative medicine because it can have a significant effect on the extent of tissue regeneration. However, biodegradation of implanted biomaterial can cause side effects on the surrounding host tissue (Sakiyama-Elbert et al., 2012). Toxic by-products might elicit immune responses or changes in the $\mathrm{pH}$, which could weaken or even reverse any beneficial treatment effects. Therefore, the efficiency of biomaterials that come into consideration as ECM mimetics should ideally be investigated both in vitro and subsequently in vivo.

The most basic function of ECMs is the provision of a scaffold structure. For neural ECM mimetics, which are used as scaffold materials in regenerative medicine, it is further desirable that they promote angiogenesis and vascularization. Blood vessels are required for the provision of nutrients and removal of waste products, 
processes that are vital for tissue regeneration (Owen and Shoichet, 2010). Angiogenesis and vascularization of the scaffold implant have been reported for numerous neural ECM biomaterials (Bakshi et al., 2004; Bramfeldt et al., 2010; Estrada et al., 2014; Plant et al., 1997; Woerly et al., 1999).

The presence and orientation of pores or channel structures in a matrix can guide the cellular growth (Sakiyama-Elbert et al., 2012). Highly complex conditions like spinal cord injuries require different molecular interventions or their combination to mediate neural cell adhesion and axon sprouting and extension. Multiple cell types and neuronal populations are affected by traumatic spinal cord injury, and therefore, possible therapeutic strategies are numerous.

Often, cells will adhere nonspecifically to unmodified ECMs. Bioengineering scaffold materials via incorporation of proteins or peptides on its surface can be used to design scaffolds with specific cell-scaffold interactions to influence the cellular behavior. Incorporation of specific peptide sequences, which promote cell adhesion, is a common way of designing functionalized ECM mimetics with the potential for enhanced cellular adhesion or better integration of the respective material (Choi et al., 2013; Dhoot et al., 2004; Plant et al., 1997).

The physical structure and rate of degradation of a neural ECM scaffold have a great impact on nervous tissue regeneration. If the material is degraded too fast, the damaged tissue may lack the necessary mechanical support that is required for regeneration. On the other hand, nondegradable materials provide a long-term structural stability but may be critical in regard to clinical applications since the implant will remain in the patient's body for an extended period of time or even permanently.

Optimal integration of ECM scaffold material will facilitate its effectiveness. It is, therefore, a high priority to minimize scarring or allergic reactions caused by the implanted material. Increased scarring responses can eventually result in an encapsulation of the foreign material from the adjacent host tissue (Sakiyama-Elbert et al., 2012; Stensaas and Stensaas, 1978). With respect to cell-matrix interaction, the hydrophobic surface of a potential neural ECM scaffold material can significantly decrease the attachment of cells (Sakiyama-Elbert et al., 2012). This effect may be desired for inflammatory cells or reactive astrocytes. But the overall prevention of cellular adhesion is an unwanted effect, which would negatively influence neural regeneration.

\section{ECM MIMETICS}

Recent advances in the design of materials, which imitate the microenvironment of the ECM, include nanofibrillar networks, artificial ECM networks, and synthetic polymers (Lutolf and Hubbell, 2005; Sadr et al., 2012; Tian et al., 2012; Tong and Yang, 2014). Depending on their designated purpose, these scaffold materials can be designed with different properties, regarding material type, ionic charge, general physicochemical properties, incorporated bioactivity (functionalization), addition of soluble factors, or biodegradability. 
The guided cell growth that is provided by such materials is intended to facilitate the structural and the functional reorganization and regeneration of dysfunctional tissues. Therapeutic approaches, which make use of matrix- or scaffold-based strategies, can be divided into two general classes (Lutolf and Hubbell, 2005):

1. Cell-based therapies

2. Acellular therapies

Cell-based therapies imply the delivery of cells and may involve the use of bioengineered tissues (biohybrid scaffolds), whereas acellular therapies rather target cells of the healthy residual tissues in situ, which can be stimulated and directed by the matrix material in their growth or differentiation.

In the field of neurodegeneration, cell transplantation strategies are widely pursued in basic research and therapy development. Although much progress has been achieved in the respective research fields, to date, it is not possible to fully restore the functions that have been lost or altered due to CNS trauma or neurodegenerative diseases. The development of scaffold materials, which serve as a matrix for transplanted cells, has advanced quite rapidly in the last years. Application of such ECM materials can promote the differentiation of stem cells into neurons and promote additional beneficial effects ( $\mathrm{Li}$ et al., 2014; Ma et al., 2008; Preston and Sherman, 2011). For cell survival and general cell behavior and function in ECM scaffold-based cell cultures and cell transplantation approaches, it is vital to incorporate additional inducers into otherwise nonbioactive materials (Mammadov et al., 2013). Ideally, for designing a suitable ECM mimetic, it is of great importance to consider both the inhibitory environment, which can result from CNS trauma or inflammation (Fawcett, 2006; Fawcett and Asher, 1999; Fitch and Silver, 2008; Klapka and Muller, 2006), and the different cues in the ECM, which significantly influence the behavior of cells in their surroundings (Chen et al., 2013; Lock et al., 2008; Ulrich et al., 2009). Such cues can be of different chemical, physical, or biological nature, or they may be triggered by cellular interactions or soluble factors. The research of the recent years has brought substantial knowledge regarding these interactions and their underlying mechanisms (Burdick and Vunjak-Novakovic, 2009; Choi et al., 2010; Engler et al., 2009; Kim et al., 2012). Therefore, the design of scaffold materials can-at least in part—be tuned to a specific purpose.

For in vitro experiments, to induce neural differentiation, the addition of soluble factors such as chemokines or growth factors to the culture medium is a common and simple approach to mimic one aspect of the interaction of cells with the ECM (Mammadov et al., 2013). The differing responses to various growth factors (neurotrophic factors, e.g., nerve growth factor (NGF), brain-derived neurotrophic factor (BDNF), and neurotrophin-3 (NT-3)) have been described for many neuronal subpopulations, and such information is advantageous for culturing the respective cells. As a strategy to mimic the neural ECM, the supply of soluble factors alone has several drawbacks, because it does not take into consideration the three-dimensional network structure of the native ECM, which is important for cellular migration and extension. Matrix materials that resemble the native ECM much more closely 
are three-dimensional scaffolding materials that can be modified further, e.g., via combination with the abovementioned soluble factors. Martino et al. (2011) had shown that the immobilization of growth factors on artificial scaffolds has a beneficial effect for tissue regeneration.

A general advantage of hydrogel ECM mimetics is their ability to develop in situ. This feature is especially useful when ECM scaffolds are required to bridge tissue defects, which are often irregularly shaped (Estrada et al., 2014; Jain et al., 2006; Sakiyama-Elbert et al., 2012). However, although hydrogels are considered as suitable ECM mimetic substrates whose porosity ensures the supply with nutrients, growth factors, and oxygen to the cell, their porous nature is also disadvantageous. Variation in stiffness causes changes in the porosity that might impair molecular diffusion in the respective scaffold material. Three-dimensional materials can generally provide the structural support, which is necessary for cellular interaction and proper cellular function because it allows the biomolecular cell-matrix interaction.

When designing ECM mimetics, it is important to consider the multiple ECM functions and the complex interaction between ECM components and cells. Model ECMs can be naturally derived or they may be synthetic materials, and they can be biodegradable or nondegradable. Materials from natural sources do have several advantages because of their inherent properties. Although, due to the generally high degree of biocompatibility of natural ECM proteins, the application of these materials appears as a reasonable approach, the results of several recent studies, however, suggest that such approaches may require additional interventions to lead to the desired effects (Banerjee et al., 2009; Engler et al., 2006; Leipzig and Shoichet, 2009). ECM mimetics are usually applied in vivo to support multicellular processes, e.g., the formation and regeneration of tissues. Moreover, functionalization of the respective material is often desired, e.g., the addition of bioactive groups such as selfassembling peptides (Maude et al., 2013) or modifications enhancing biodegradability (Liu et al., 2012).

Bioengineered ECM scaffolds can be designed for the controlled release of proteins. In diffusion-based delivery systems, the amount of the released compound can be regulated by physical characteristics, such as the pore size or the degree of porosity, or by the degradation rate of the material (Houweling et al., 1998; SakiyamaElbert et al., 2012). Drug delivery can also be achieved by affinity-based systems (Taylor et al., 2006), which allow the controlled release of a substance. An additional possibility is the covalent attachment of the compound to the material (Tian et al., 2005). In this case, the release will occur solely via the degradation of the scaffold material. Such manipulation by modulating physicochemical properties or adding functionalized bioactive groups is a challenge, which is generally more successful in synthetic materials rather than natural matrices.

Furthermore, purification, immunogenicity, and pathogen transmission are important issues that must be considered for ECM-based approaches, and regarding such issues, the design of synthetic materials presents a well-controllable option. Some of the most common natural and synthetic materials that have recently been used as ECM mimetics are described below. 


\section{BIOMEDICAL NEURAL ECM MIMETICS 5.1 NATURALLY DERIVED ECM MIMETICS}

For many in vitro cell culture experiments, two-dimensional assays are still the method of choice. However, the generally accepted opinion is that three-dimensional matrices are the preferable ECM model systems because they mimic the physiological situation of in vivo tissues more closely (Lutolf and Hubbell, 2005; Mammadov et al., 2013; Owen and Shoichet, 2010). Examples for ECM models of natural sources are matrices such as Matrigel ${ }^{\mathrm{TM}}$ (a basement membrane preparation derived from mouse tumor tissue), matrices that consist of individual ECM components, such as collagen, fibrin, or fibronectin, and fragmented or modified ECM components. Natural ECMs are gels that consist of fibrous and fibrillary proteins within a hydrated network of GAG chains. Their structural architecture and their inherent biophysical properties can have significant effects on cellular functions, e.g., adhesion and migration. Naturally derived neural ECM mimetics have been used in therapeutic strategies mostly either as cell carriers for tissue grafting or as regeneration-promoting matrices that are implanted to bridge tissue defects.

\subsubsection{Collagen}

Collagen plays a dual role for neural growth and regeneration in vivo. It is the most abundant protein of native ECMs, and its suitability as a growth-promoting scaffold material has been studied extensively. Collagen's inherent integrin binding sites are advantageous as they promote the migration and differentiation of neuronal cells (Bradshaw et al., 1995). Modification of collagen scaffolds via the addition of neurotrophic factors (Han et al., 2009; Houweling et al., 1998), other pharmacologically active substances (Bolliet et al., 2008), or cells (Joosten et al., 2004) has been described to increase axonal regeneration and functional recovery after spinal cord trauma. Despite the fact that collagen per se is a suitable substrate for neuronal growth, it is also important to consider the role of collagen as the basement membrane constituent of the lesion scar that develops after a traumatic CNS injury (Fawcett et al., 2012; Klapka and Muller, 2006). The collagen matrix of the lesion scar is a sticky matrix to which numerous growth-inhibitory molecules that are upregulated after injury can adhere to and thereby impede neuronal growth.

\subsubsection{HA-Derived Materials}

HA is a nonimmunogenic linear GAG that is a constitutive of soft connective tissue and is involved in wound healing. It has been reported that HA implantation reduces scar formation in both PNS and CNS (Wang et al., 2012). In this context, its inhibitory effect on the activation of astrocytes, CSPG deposition, and infiltration of macrophages/microglia appears to be dependent on its molecular weight (Campo et al., 2010; Khaing et al., 2011). A disadvantage of HA is that cells do not attach to its surface (Wang et al., 2012). However, its combination with other scaffold materials or functionalization with peptide sequences or other bioactive inducers has been 
described to result in suitable mechanical properties for CNS regeneration in vivo (Wang and Spector, 2009) and promote neuronal differentiation in vitro (Brannvall et al., 2007).

\subsubsection{Alginate}

Alginate is a water-soluble anionic polysaccharide distributed widely in the cell walls of brown algae, where it forms a viscous gum through binding water. Alginate is a linear copolymer with homopolymeric blocks of (1-4)-linked $\beta$-D-mannuronate (M) and its C-5 epimer $\alpha$-L-guluronate $(\mathrm{G})$ residues, respectively, covalently linked together in different sequences or blocks. Alginate is immunologically inert and is not digested by mammalian cells. As the cross-linking multivalent cations gradually diffuse out from the gel, it slowly degrades and is excreted in urine. Alginate gels are frequently used as matrices for encapsulation of living cells and for the release of proteins (Novikova et al., 2006; Tobias et al., 2001, 2005). In regeneration studies, alginate gels are often applied as cell carrier substances. Alginate has been described to enhance neuronal sprouting and to decrease inhibitory cues after CNS trauma (Kataoka et al., 2004; Prang et al., 2006), but other studies have also reported a lack of axon growth stimulation mediated by alginate scaffolds (Estrada et al., 2014; Novikova et al., 2006). In vitro studies have even demonstrated that this biomaterial can inhibit the growth of dorsal root ganglion neurons and cause alteration of the phenotype of various different cell types (Novikova et al., 2006). This gel is, however, a quite suitable matrix for the encapsulation of cells with or even without further modification of the material.

\subsubsection{Agarose}

The linear polysaccharide agarose is generally well tolerated after its implantation (Tashiro et al., 1997). Although its mechanical properties can be modified to mimic the mechanical properties of its host tissue (Balgude et al., 2001), an agarose ECM mimetic alone-when compared with the effectivity of other natural ECM mimeticsdoes not suffice to achieve significant degrees of neuronal growth (Lin et al., 2005). Via modification of the material prior to implantation, the neuronal growth rate through agarose scaffolds can, however, be increased (Gros et al., 2010; Jain et al., 2006; Lee et al., 2010; Sakiyama-Elbert et al., 2012; Stokols and Tuszynski, 2006).

\subsubsection{Matrige $^{\mathrm{TM}}$}

Matrigel $^{\mathrm{TM}}$ is a gelatinous ECM protein mixture obtained from the murine EngelbrethHolm-Swarm sarcoma. It comprises a mixture of Col4, laminin, and heparan sulfate proteoglycan admixed with other minor amounts of extracellular components, as well as growth factors. As a biodegradable implant, Matrigel ${ }^{\mathrm{TM}}$ resembles the complex extracellular environment found in many healthy tissues (Kleinman and Martin, 2005) and is widely used as a substrate for cell culture, which forms a nonporous hydrogel at physiological temperature. It has been described as a suitable matrix for tissue generation (Cassell et al., 2001) and has led to neuronal regeneration of varying degrees from only limited growth to extensive axonal sprouting in several studies (Iannotti 
et al., 2003; Novikova et al., 2006). Scaffolds of Matrigel ${ }^{\mathrm{TM}}$ allow only limited axonal growth after spinal cord trauma (Estrada et al., 2014; Sakiyama-Elbert et al., 2012; Someya et al., 2008). This ECM mimetic, however, seems to exert significant beneficial, growth-promoting effects when it is used as a cell carrier medium in cell transplantation studies. In combination with cellular transplants, especially Schwann cells, Matrigel $^{\mathrm{TM}}$ is a widely used biomaterial for neuronal regeneration, where it has been shown to obtain positive results regarding axonal regeneration after central and peripheral nerve injury (Fouad et al., 2005; Novikova et al., 2006; Rodriguez et al., 2000; Someya et al., 2008; Xu et al., 1995, 1997). In contrast to alginate, Matrigel ${ }^{\mathrm{TM}}$ has not been found to alter the cellular morphology of olfactory ensheathing glia, Schwann cells, or bone marrow stem cells, and it has been reported to stimulate the growth of dorsal root ganglia in vitro (Novikova et al., 2006). Despite reported beneficial effects, the clinical use of Matrigel ${ }^{\mathrm{TM}}$ remains questionable because of its tumor-derived origin and its batch-to-batch variability (Sakiyama-Elbert et al., 2012). Batch variation, which might significantly influence the effectiveness, is, in fact, a common disadvantage of natural biomaterials when applied as ECM scaffolds (Orive et al., 2006; Owen and Shoichet, 2010; Wang et al., 2012).

\subsubsection{Chitosan}

The biodegradable and nontoxic chitosan can be modified to form scaffolds, which contain pores or channels that facilitate neuronal growth (Li et al., 2009). Chitosan is produced via deacetylation of chitin. The manipulation of chitosan and the resulting degree of deacetylation have been reported to influence the adhesion and migration behavior of cells on the surface of the matrix (Chatelet et al., 2001). However, the growth-promoting effects of chitosan scaffolds can be further enhanced via the introduction of functionalized groups or combination with growth factors (Chen et al., 2011; Cheng et al., 2007; Goraltchouk et al., 2006; Nomura et al., 2008; Yu et al., 2007).

\subsubsection{Fibrin}

The fibrous protein fibrin is involved in the clotting of blood during wound healing. During this process of tissue repair, fibrin molecules form an ECM meshwork, which stimulates cellular proliferation and migration. Two RGD recognition sequences provide binding sites for integrin receptors, which mediate cell adhesion and can significantly influence cellular migration or growth processes (Mosesson, 2005). The use of fibrin scaffolds is safe, lacking side effects. And manipulation of fibrin to create delivery systems that can be used for controlled release of bioactive factors has been tested extensively and yielded positive effects ( Johnson et al., 2010; SakiyamaElbert et al., 2012; Taylor et al., 2006).

\subsubsection{Fibronectin}

Fibronectin is a key effector of fibrosis. As a globular plasma protein, it exhibits its soluble form, and as an ECM protein, it takes on a fibrous insoluble form. Via its binding to integrins through specific peptide sequences, it is strongly involved in adhesion-mediated cellular migration (Iannotti et al., 2003). After CNS trauma, 
fibronectin ECM mimetics exert growth-promoting and guiding effects on spinal axons (King et al., 2003; Phillips et al., 2004). Additionally, the promotion of cellular infiltration of fibronectin scaffolds with regeneration-supporting glial cells has been described (King et al., 2006).

\subsection{SYNTHETIC ECM MIMETICS}

Synthetic scaffold materials are generally developed with the intention to fabricate matrices with structural characteristics that resemble those of the native ECM. Although two-dimensional fibrous or fibrillary substrates may result in deviant cellular shapes and behavior, they have proven quite successful in neural tissue engineering approaches where they have been demonstrated to facilitate CNS regeneration (Lutolf and Hubbell, 2005).

The design of synthetic ECMs offers several advantages: bioengineering technologies allow the fabrication of fibrillar biomaterials of nanometer-scale dimensions via polymer processing or supramolecular self-assembly. The typical hydrogel character of native ECM can be physicochemically mimicked by synthetic hydrogels. Synthetic hydrogels can often be formed in situ, which makes their application especially attractive for cell-containing hydrogels, which require gentle experimental protocols (Lutolf and Hubbell, 2005).

The possibility to incorporate bioactive signaling cues in both fibrillary- and hydrogel-based neural ECM renders them to be very useful scaffold materials for tissue regeneration.

Both biodegradable and nondegradable synthetic matrices can be fabricated, and their application has been investigated extensively.

\subsubsection{Polyethylene Glycol}

Polyethylene glycol (PEG) is a fusogen agent, which has been demonstrated to reunite transected cell processes and seal cell membranes after mechanical spinal insult (Borgens, 2001; Borgens and Bohnert, 2001; Borgens and Shi, 2000; Borgens et al., 2002). Acute PEG treatment after spinal cord trauma has also been reported to exert neuroprotective effects via the reduction of oxidative stress reactions (Luo and Shi, 2004; Luo et al., 2004). PEG is a widely used material for the design of biodegradable synthetic cross-linked hydrogels (Burdick et al., 2006; Cong et al., 2009; Gunn et al., 2005; Herten et al., 2009; Phelps et al., 2010; Qiao et al., 2005; Raeber et al., 2005; Rooney et al., 2011). PEG has several beneficial features, which are advantageous for soft tissue regeneration. PEG hydrogels are hydrophilic polymers that generally contain cross-links that exert high degrees of swelling in aqueous environments such as soft tissues (Hoffman, 2002; Lee and Mooney, 2001). PEG is biocompatible and PEG hydrogels are generally not prone to nonspecific cell adhesion or protein adsorption. Such features provide an ideal basis for the introduction of ECM-derived signals, which can stimulate the regeneration of tissues and cells (Chung et al., 2008). Although PEG itself is a material that cells generally do not attach to (Cong et al., 2009), bioengineered PEG hydrogels can also be used as a matrix for the delivery of cells. 
Although the most common approaches using PEG for ECM mimetics involve the bioengineering of PEG-containing hydrogels, the generally inert, nontoxic, and nonimmunogenic nature of PEG can also be made use of in a PEG polymer matrix. Beneficial effects of a PEG biopolymer matrix have been described recently. In the respective study, a solution of pure PEG 600 was applied after resection of spinal scar tissue in rats with severe chronic spinal cord injury (Estrada et al., 2014). Although PEG polymers per se do not conform to the basic requirement of an ECM mimetic, which is to provide structural support, they nevertheless promote the formation of a beneficial stable microenvironment in vivo. The PEG application promoted the infiltration with cells, which are beneficial for the regeneration of tissue in general and axons in particular. At the same time, the degree of reformation of scar tissue was decreased, which allowed the development of a stable biomatrix at the site of resection. Revascularization, invasion of astrocytes and Schwann cells, and the formation of a stable ECM-containing biopolymer were reported. Furthermore, such PEG treatment alone was sufficient to result in the myelination of regenerated axons and significant locomotor functional improvements in the treated animals (Estrada et al., 2014). Since the chemical properties of PEGs with different molecular weights are nearly identical, the positive treatment effects in the latter study were attributed to the physical properties of the applied PEG (PEG 600): while the tested lower-molecular-weight PEG was too fluid to allow the material to remain at the resection site, the tested PEG of a higher molecular weight even leads to an encapsulation of the implanted material. Only PEG 600-a PEG with a low viscosity-promoted the soft tissue regeneration in the treated area that resulted in the observed cellular invasion and axonal elongation. Such findings argue for the importance of the appropriate stiffness/viscosity that needs to be considered for a respective application.

\subsubsection{Lactide- and Glycolide-Derived Polyesters}

Poly(lactic acid), poly(glycolic acid), and their copolymers poly(lactic-co-glycolic acid) are widely used biomaterials for tissue engineering strategies. They are biocompatible and biodegradable, and their mechanophysical properties (and thus the rate and timing of degradation) can be modulated to some degree (SakiyamaElbert et al., 2012). However, several studies have reported undesired effects or a lack of success that might be explained by the rate of degradation and a loss of structural support (Deumens et al., 2006; Hurtado et al., 2006).

\subsubsection{Polycaprolactones}

Polycaprolactones are biodegradable polymers, which can be used to fabricate threedimensional tubular structures with varying degrees of porosity. Implantation of combinations of polycaprolactones with other polysaccharide-based hydrogel materials has been described to reduce inflammation responses after spinal cord injury (Silva et al., 2010). Poly- $\varepsilon$-caprolactone can be used for three-dimensional bioprinting, which makes it a very promising material for novel personalized medical approaches (Wong et al., 2008). 


\subsubsection{Poly(2-hydroxyethyl Methacrylate)}

Poly(2-hydroxyethyl methacrylate) is a nontoxic, nonbiodegradable hydrogel. The material allows the attachment and growth of cells and the transport of small molecules. It can also serve as a guidance substrate for neurons as has been shown for spinal cord trauma (Hejcl et al., 2008). The nonbiodegradable nature can be considered as both advantageous and unfavorable: It ensures the maintenance of a stable scaffold structure over time. At the same time, especially in regard to its clinical application, the scaffold will remain in the patient's body after the implantation. As a foreign substance, it might cause side effects or unpredictable long-term consequences.

\subsubsection{NeuroGel $^{\mathrm{TM}}$}

NeuroGel $^{\mathrm{TM}}$ (N-(2-hydroxypropyl)methacrylamide or HPMA) is another nonbiodegradable hydrogel that has been described to support cellular infiltration, angiogenesis, and axonal growth after CNS trauma (Woerly, 2000; Woerly et al., 2001). The surface of this scaffold can also be chemically modified via incorporation of bioactive sequences to increase its beneficial effects.

\subsubsection{Nanostructured Materials}

Nanotechnology offers an enormous potential for the fabrication of nanostructures (particles and fibers). Via electrospinning, nanofiber scaffolds that can be used for cell culturing can be fabricated. Electrospun polyurethane scaffolds with high porosity were previously shown to induce differentiation of hESCs into neurons (Carlberg et al., 2009). In another study, PC12 cells seeded on NGF encapsulated electrospun copolymer of $\varepsilon$-caprolactone and ethyl ethylene phosphate scaffolds were observed to exhibit enhanced neurite outgrowth (Chew et al., 2005). Chemical conjugation to electrospun nanofibers is also effective in inducing neural differentiation. NGF-conjugated aligned electrospun PEG-poly( $\varepsilon$-caprolactone) nanofibers induced transdifferentiation of MSCs into neural cells after 7 days (Cho et al., 2010).

\subsubsection{Self-assembling Materials}

Self-assembling nanofibers can be tuned in such a way that they form scaffold structures when injected into neural tissue (Berns et al., 2014). Self-assembling scaffolds can be functionalized by using peptide sequences from ECM proteins or other neural differentiation inducers that bind to cell surface receptors. Peptide amphiphiles that self-assemble into nanofibers have successfully been used for neural differentiation in vitro and for in vivo bridging of CNS tissue defects in several studies (Mammadov et al., 2012a,b; Silva et al., 2004; Tysseling et al., 2010; Tysseling-Mattiace et al., 2008). Self-assembled peptide nanofibers produced from alternating basic, hydrophobic, and acidic amino acids (RADA16) have also been shown to enhance neural cell culture and provide therapeutic effects in several CNS dysfunctions (EllisBehnke et al., 2006; Gelain et al., 2011; Guo et al., 2009; Holmes et al., 2000; Silva, 2005). Composites of peptides and nanofibers are very promising scaffold materials for neural growth and regeneration. The incorporation of self-assembling 
nanofibers into other matrices makes them very suitable also for cell transplantationbased approaches (Romano et al., 2011).

Generally, nerve grafts - the conventional but limited standard to treat neural defects - have been replaced by the development of novel synthetic scaffolds. The selected list of biomaterials described here covers only a portion of the numerous potential ECM mimetic scaffolds. Recent advances in tissue engineering and nanotechnology demonstrate that neural replacement and repair can be achieved to a remarkable extent. The intensive research regarding development and application of neural ECM mimetics shows that a major determinant is the regulation of cellular behavior by mimicking native ECM features. Desired material properties of neural ECM are mechanical stability, biocompatibility with low or even absence of immunogenicity, controlled biodegradability, and a structure that allows cell migration (porosity) and vascularization. Current technological and engineering methods allow highly specialized designs, which most likely will lead to rapid advances in regenerative medicine in the future.

\section{LOST IN TRANSLATION}

The replication of the native neural ECM environment is a major challenge. The quest for suitable scaffold materials has led to a better understanding of ECM-mediated functions and has encouraged the development and optimization of suitable bioengineering technologies. The ECM mimetic materials mentioned above only represent a selection of the multitude of biomaterials, which can be used as neural ECM scaffolds. A suitable ECM mimetic needs to exert the principal functions of the native ECM: providing a structural scaffold and mediating cell proliferation, migration, differentiation, survival, regeneration, angiogenesis, and invasion. The orchestration of these cellular processes requires a functional network of biochemical and structural cues.

An important criterion for the design and application of a biomimetic ECM is its translational potential. Regarding the translation from in vitro experiments to the in vivo development of therapies in large animal models and into clinical applications, it is necessary to prove the effectiveness of a material and its suitability for the intended purpose both in vitro and in vivo. Moreover and very importantly, safety of the material needs to be demonstrated prior to its application into patients.

Due to the number and complexity of physiological functions, which are mediated by the native ECM, a single formulation cannot meet the requirements for every task (Prestwich, 2008), from cell expansion and delivery to providing a stable scaffold structure and from cell replacement strategies to large-scale repair of tissue defects and regeneration-promoting therapies. For a translation from in vitro experiments to clinical applications, it is, therefore, important to optimize the design of ECM mimetic materials with high degrees of flexibility, which would allow the use of few suitable materials for multiple purposes (Prestwich, 2008). 


\section{ACKNOWLEDGMENTS}

The authors would like to thank Dr. Bernhard Wehrle-Haller for his critical reading of the manuscript and COST Action ECMNET for making this publication possible.

\section{REFERENCES}

Bakshi, A., Fisher, O., Dagci, T., Himes, B.T., Fischer, I., Lowman, A., 2004. Mechanically engineered hydrogel scaffolds for axonal growth and angiogenesis after transplantation in spinal cord injury. J. Neurosurg. Spine 1, 322-329.

Balgude, A.P., Yu, X., Szymanski, A., Bellamkonda, R.V., 2001. Agarose gel stiffness determines rate of DRG neurite extension in 3D cultures. Biomaterials 22, 1077-1084.

Banerjee, A., Arha, M., Choudhary, S., Ashton, R.S., Bhatia, S.R., Schaffer, D.V., Kane, R.S., 2009. The influence of hydrogel modulus on the proliferation and differentiation of encapsulated neural stem cells. Biomaterials 30, 4695-4699.

Benowitz, L.I., Popovich, P.G., 2011. Inflammation and axon regeneration. Curr. Opin. Neurol. 24, 577-583.

Berns, E.J., Sur, S., Pan, L., Goldberger, J.E., Suresh, S., Zhang, S., Kessler, J.A., Stupp, S.I., 2014. Aligned neurite outgrowth and directed cell migration in self-assembled monodomain gels. Biomaterials 35, 185-195.

Bolliet, C., Bohn, M.C., Spector, M., 2008. Non-viral delivery of the gene for glial cell linederived neurotrophic factor to mesenchymal stem cells in vitro via a collagen scaffold. Tissue Eng. Part C Methods 14, 207-219.

Borgens, R.B., 2001. Cellular engineering: molecular repair of membranes to rescue cells of the damaged nervous system. Neurosurgery 49, 370-378.

Borgens, R.B., Bohnert, D., 2001. Rapid recovery from spinal cord injury after subcutaneously administered polyethylene glycol. J. Neurosci. Res. 66, 1179-1186.

Borgens, R.B., Shi, R., 2000. Immediate recovery from spinal cord injury through molecular repair of nerve membranes with polyethylene glycol. FASEB J. 14, 27-35.

Borgens, R.B., Shi, R., Bohnert, D., 2002. Behavioral recovery from spinal cord injury following delayed application of polyethylene glycol. J. Exp. Biol. 205 (Pt 1), 1-12.

Bradshaw, A.D., McNagny, K.M., Gervin, D.B., Cann, G.M., Graf, T., Clegg, D.O., 1995. Integrin alpha 2 beta 1 mediates interactions between developing embryonic retinal cells and collagen. Development 121, 3593-3602.

Bramfeldt, H., Sabra, G., Centis, V., Vermette, P., 2010. Scaffold vascularization: a challenge for three-dimensional tissue engineering. Curr. Med. Chem. 17, 3944-3967.

Brannvall, K., Bergman, K., Wallenquist, U., Svahn, S., Bowden, T., Hilborn, J., ForsbergNilsson, K., 2007. Enhanced neuronal differentiation in a three-dimensional collagenhyaluronan matrix. J. Neurosci. Res. 85, 2138-2146.

Burdick, J.A., Vunjak-Novakovic, G., 2009. Engineered microenvironments for controlled stem cell differentiation. Tissue Eng. Part A 15, 205-219.

Burdick, J.A., Ward, M., Liang, E., Young, M.J., Langer, R., 2006. Stimulation of neurite outgrowth by neurotrophins delivered from degradable hydrogels. Biomaterials 27, 452-459. 
Campo, G.M., Avenoso, A., Campo, S., D’Ascola, A., Nastasi, G., Calatroni, A., 2010. Molecular size hyaluronan differently modulates toll-like receptor-4 in LPS-induced inflammation in mouse chondrocytes. Biochimie 92, 204-215.

Carlberg, B., Axell, M.Z., Nannmark, U., Liu, J., Kuhn, H.G., 2009. Electrospun polyurethane scaffolds for proliferation and neuronal differentiation of human embryonic stem cells. Biomed. Mater. 4, 045004.

Cartier, L., Hartley, O., Dubois-Dauphin, M., Krause, K.H., 2005. Chemokine receptors in the central nervous system: role in brain inflammation and neurodegenerative diseases. Brain Res. Brain Res. Rev. 48, 16-42.

Cassell, O.C., Morrison, W.A., Messina, A., Penington, A.J., Thompson, E.W., Stevens, G.W., Perera, J.M., Kleinman, H.K., Hurley, J.V., Romeo, R., Knight, K.R., 2001. The influence of extracellular matrix on the generation of vascularized, engineered, transplantable tissue. Ann. N.Y. Acad. Sci. 944, 429-442.

Chatelet, C., Damour, O., Domard, A., 2001. Influence of the degree of acetylation on some biological properties of chitosan films. Biomaterials 22, 261-268.

Chen, X., Yang, Y., Yao, J., Lin, W., Li, Y., Chen, Y., Gao, Y., Yang, Y., Gu, X., Wang, X., 2011. Bone marrow stromal cells-loaded chitosan conduits promote repair of complete transection injury in rat spinal cord. J. Mater. Sci. Mater. Med. 22, 2347-2356.

Chen, Y., Dodd, S.J., Tangrea, M.A., Emmert-Buck, M.R., Koretsky, A.P., 2013. Measuring collective cell movement and extracellular matrix interactions using magnetic resonance imaging. Sci. Rep. 3, 1879.

Cheng, H., Huang, Y.C., Chang, P.T., Huang, Y.Y., 2007. Laminin-incorporated nerve conduits made by plasma treatment for repairing spinal cord injury. Biochem. Biophys. Res. Commun. 357, 938-944.

Chew, S.Y., Wen, J., Yim, E.K., Leong, K.W., 2005. Sustained release of proteins from electrospun biodegradable fibers. Biomacromolecules 6, 2017-2024.

Cho, Y.I., Choi, J.S., Jeong, S.Y., Yoo, H.S., 2010. Nerve growth factor (NGF)-conjugated electrospun nanostructures with topographical cues for neuronal differentiation of mesenchymal stem cells. Acta Biomater. 6, 4725-4733.

Choi, C.K., Breckenridge, M.T., Chen, C.S., 2010. Engineered materials and the cellular microenvironment: a strengthening interface between cell biology and bioengineering. Trends Cell Biol. 20, 705-714.

Choi, S.K., Park, J.K., Lee, K.M., Lee, S.K., Jeon, W.B., 2013. Improved neural progenitor cell proliferation and differentiation on poly(lactide-co-glycolide) scaffolds coated with elastin-like polypeptide. J. Biomed. Mater. Res. B Appl. Biomater. 101, 1329-1339.

Chung, I.M., Enemchukwu, N.O., Khaja, S.D., Murthy, N., Mantalaris, A., Garcia, A.J., 2008. Bioadhesive hydrogel microenvironments to modulate epithelial morphogenesis. Biomaterials 29, 2637-2645.

Cong, H., Revzin, A., Pan, T., 2009. Non-adhesive PEG hydrogel nanostructures for selfassembly of highly ordered colloids. Nanotechnology 20, 075307.

David, S., Aguayo, A.J., 1981. Axonal elongation into peripheral nervous system "bridges" after central nervous system injury in adult rats. Science 214, 931-933.

Deumens, R., Koopmans, G.C., Honig, W.M., Hamers, F.P., Maquet, V., Jerome, R., Steinbusch, H.W., Joosten, E.A., 2006. Olfactory ensheathing cells, olfactory nerve fibroblasts and biomatrices to promote long-distance axon regrowth and functional recovery in the dorsally hemisected adult rat spinal cord. Exp. Neurol. 200, 89-103. 


\section{CHAPTER 16 Neural ECM mimetics}

Dhoot, N.O., Tobias, C.A., Fischer, I., Wheatley, M.A., 2004. Peptide-modified alginate surfaces as a growth permissive substrate for neurite outgrowth. J. Biomed. Mater. Res. 71A, 191-200.

Ellis-Behnke, R.G., Liang, Y.X., You, S.W., Tay, D.K., Zhang, S., So, K.F., Schneider, G.E., 2006. Nano neuro knitting: peptide nanofiber scaffold for brain repair and axon regeneration with functional return of vision. Proc. Natl. Acad. Sci. U. S. A. 103, 5054-5059.

Engler, A.J., Sen, S., Sweeney, H.L., Discher, D.E., 2006. Matrix elasticity directs stem cell lineage specification. Cell 126, 677-689.

Engler, A.J., Humbert, P.O., Wehrle-Haller, B., Weaver, V.M., 2009. Multiscale modeling of form and function. Science 324, 208-212.

Estrada, V., Brazda, N., Schmitz, C., Heller, S., Blazyca, H., Martini, R., Muller, H.W., 2014. Long-lasting significant functional improvement in chronic severe spinal cord injury following scar resection and polyethylene glycol implantation. Neurobiol. Dis. 67C, $165-179$.

Fawcett, J.W., 2006. Overcoming inhibition in the damaged spinal cord. J. Neurotrauma $23,371-383$.

Fawcett, J.W., Asher, R.A., 1999. The glial scar and central nervous system repair. Brain Res. Bull. 49, 377-391.

Fawcett, J.W., Schwab, M.E., Montani, L., Brazda, N., Muller, H.W., 2012. Defeating inhibition of regeneration by scar and myelin components. Handb. Clin. Neurol. 109, 503-522.

Ferguson, T.A., Son, Y.J., 2011. Extrinsic and intrinsic determinants of nerve regeneration. J. Tissue Eng. 2, 2041731411418392.

Fitch, M.T., Silver, J., 2008. CNS injury, glial scars, and inflammation: inhibitory extracellular matrices and regeneration failure. Exp. Neurol. 209, 294-301.

Fouad, K., Schnell, L., Bunge, M.B., Schwab, M.E., Liebscher, T., Pearse, D.D., 2005. Combining Schwann cell bridges and olfactory-ensheathing glia grafts with chondroitinase promotes locomotor recovery after complete transection of the spinal cord. J. Neurosci. 25 (5), $1169-1178$.

Gelain, F., Panseri, S., Antonini, S., Cunha, C., Donega, M., Lowery, J., Taraballi, F., Cerri, G., Montagna, M., Baldissera, F., Vescovi, A., 2011. Transplantation of nanostructured composite scaffolds results in the regeneration of chronically injured spinal cords. ACS Nano 5, 227-236.

Goraltchouk, A., Scanga, V., Morshead, C.M., Shoichet, M.S., 2006. Incorporation of proteineluting microspheres into biodegradable nerve guidance channels for controlled release. J. Control. Release 110, 400-407.

Gros, T., Sakamoto, J.S., Blesch, A., Havton, L.A., Tuszynski, M.H., 2010. Regeneration of long-tract axons through sites of spinal cord injury using templated agarose scaffolds. Biomaterials 31, 6719-6729.

Gunn, J.W., Turner, S.D., Mann, B.K., 2005. Adhesive and mechanical properties of hydrogels influence neurite extension. J. Biomed. Mater. Res. A 72, 91-97.

Guo, J., Leung, K.K., Su, H., Yuan, Q., Wang, L., Chu, T.H., Zhang, W., Pu, J.K., Ng, G.K., Wong, W.M., Dai, X., Wu, W., 2009. Self-assembling peptide nanofiber scaffold promotes the reconstruction of acutely injured brain. Nanomedicine 5, 345-351.

Han, Q., Sun, W., Lin, H., Zhao, W., Gao, Y., Zhao, Y., Chen, B., Xiao, Z., Hu, W., Li, Y., Yang, B., Dai, J., 2009. Linear ordered collagen scaffolds loaded with collagen-binding brain-derived neurotrophic factor improve the recovery of spinal cord injury in rats. Tissue Eng. Part A 15, 2927-2935. 
Hejcl, A., Urdzikova, L., Sedy, J., Lesny, P., Pradny, M., Michalek, J., Burian, M., Hajek, M., Zamecnik, J., Jendelova, P., Sykova, E., 2008. Acute and delayed implantation of positively charged 2-hydroxyethyl methacrylate scaffolds in spinal cord injury in the rat. J. Neurosurg. Spine 8, 67-73.

Herten, M., Jung, R.E., Ferrari, D., Rothamel, D., Golubovic, V., Molenberg, A., Hammerle, C.H., Becker, J., Schwarz, F., 2009. Biodegradation of different synthetic hydrogels made of polyethylene glycol hydrogel/RGD-peptide modifications: an immunohistochemical study in rats. Clin. Oral Implants Res. 20, 116-125.

Hoffman, A.S., 2002. Hydrogels for biomedical applications. Adv. Drug Deliv. Rev. 54, 3-12.

Holmes, T.C., de Lacalle, S., Su, X., Liu, G., Rich, A., Zhang, S., 2000. Extensive neurite outgrowth and active synapse formation on self-assembling peptide scaffolds. Proc. Natl. Acad. Sci. U. S. A. 97, 6728-6733.

Houweling, D.A., Lankhorst, A.J., Gispen, W.H., Bar, P.R., Joosten, E.A., 1998. Collagen containing neurotrophin-3 (NT-3) attracts regrowing injured corticospinal axons in the adult rat spinal cord and promotes partial functional recovery. Exp. Neurol. 153, 49-59.

Hurtado, A., Moon, L.D., Maquet, V., Blits, B., Jerome, R., Oudega, M., 2006. Poly (D, L-lactic acid) macroporous guidance scaffolds seeded with Schwann cells genetically modified to secrete a bi-functional neurotrophin implanted in the completely transected adult rat thoracic spinal cord. Biomaterials 27, 430-442.

Iannotti, C., Li, H., Yan, P., Lu, X., Wirthlin, L., Xu, X.M., 2003. Glial cell line-derived neurotrophic factor-enriched bridging transplants promote propriospinal axonal regeneration and enhance myelination after spinal cord injury. Exp. Neurol. 183, 379-393.

Jain, A., Kim, Y.T., McKeon, R.J., Bellamkonda, R.V., 2006. In situ gelling hydrogels for conformal repair of spinal cord defects, and local delivery of BDNF after spinal cord injury. Biomaterials 27, 497-504.

Johnson, P.J., Parker, S.R., Sakiyama-Elbert, S.E., 2010. Fibrin-based tissue engineering scaffolds enhance neural fiber sprouting and delay the accumulation of reactive astrocytes at the lesion in a subacute model of spinal cord injury. J. Biomed. Mater. Res. A 92, 152-163.

Joosten, E.A., Veldhuis, W.B., Hamers, F.P., 2004. Collagen containing neonatal astrocytes stimulates regrowth of injured fibers and promotes modest locomotor recovery after spinal cord injury. J. Neurosci. Res. 77, 127-142.

Kataoka, K., Suzuki, Y., Kitada, M., Hashimoto, T., Chou, H., Bai, H., Ohta, M., Wu, S., Suzuki, K., Ide, C., 2004. Alginate enhances elongation of early regenerating axons in spinal cord of young rats. Tissue Eng. 10, 493-504.

Khaing, Z.Z., Milman, B.D., Vanscoy, J.E., Seidlits, S.K., Grill, R.J., Schmidt, C.E., 2011. High molecular weight hyaluronic acid limits astrocyte activation and scar formation after spinal cord injury. J. Neural Eng. 8, 046033.

Kim, D.H., Provenzano, P.P., Smith, C.L., Levchenko, A., 2012. Matrix nanotopography as a regulator of cell function. J. Cell Biol. 197, 351-360.

King, V.R., Henseler, M., Brown, R.A., Priestley, J.V., 2003. Mats made from fibronectin support oriented growth of axons in the damaged spinal cord of the adult rat. Exp. Neurol. 182, 383-398.

King, V.R., Phillips, J.B., Hunt-Grubbe, H., Brown, R., Priestley, J.V., 2006. Characterization of non-neuronal elements within fibronectin mats implanted into the damaged adult rat spinal cord. Biomaterials 27, 485-496.

Klapka, N., Muller, H.W., 2006. Collagen matrix in spinal cord injury. J. Neurotrauma 23, 422-435. 
Kleinman, H.K., Martin, G.R., 2005. Matrigel: basement membrane matrix with biological activity. Semin. Cancer Biol. 15, 378-386.

Lee, K.Y., Mooney, D.J., 2001. Hydrogels for tissue engineering. Chem. Rev. 101, 1869-1879.

Lee, H., McKeon, R.J., Bellamkonda, R.V., 2010. Sustained delivery of thermostabilized chABC enhances axonal sprouting and functional recovery after spinal cord injury. Proc. Natl. Acad. Sci. U. S. A. 107, 3340-3345.

Leipzig, N.D., Shoichet, M.S., 2009. The effect of substrate stiffness on adult neural stem cell behavior. Biomaterials 30, 6867-6878.

Li, X., Yang, Z., Zhang, A., 2009. The effect of neurotrophin-3/chitosan carriers on the proliferation and differentiation of neural stem cells. Biomaterials 30, 4978-4985.

Li, Y., Liu, M., Yan, Y., Yang, S.T., 2014. Neural differentiation from pluripotent stem cells: the role of natural and synthetic extracellular matrix. World J. Stem Cells 6, 11-23.

Lin, P.W., Wu, C.C., Chen, C.H., Ho, H.O., Chen, Y.C., Sheu, M.T., 2005. Characterization of cortical neuron outgrowth in two- and three-dimensional culture systems. J. Biomed. Mater. Res. B Appl. Biomater. 75, 146-157.

Liu, X., Holzwarth, J.M., Ma, P.X., 2012. Functionalized synthetic biodegradable polymer scaffolds for tissue engineering. Macromol. Biosci. 12, 911-919.

Lock, J.G., Wehrle-Haller, B., Stromblad, S., 2008. Cell-matrix adhesion complexes: master control machinery of cell migration. Semin. Cancer Biol. 18, 65-76.

Luo, J., Shi, R., 2004. Diffusive oxidative stress following acute spinal cord injury in guinea pigs and its inhibition by polyethylene glycol. Neurosci. Lett. 359, 167-170.

Luo, J., Borgens, R., Shi, R., 2004. Polyethylene glycol improves function and reduces oxidative stress in synaptosomal preparations following spinal cord injury. J. Neurotrauma 21, 994-1007.

Lutolf, M.P., Hubbell, J.A., 2005. Synthetic biomaterials as instructive extracellular microenvironments for morphogenesis in tissue engineering. Nat. Biotechnol. 23, 47-55.

Ma, W., Tavakoli, T., Derby, E., Serebryakova, Y., Rao, M.S., Mattson, M.P., 2008. Cellextracellular matrix interactions regulate neural differentiation of human embryonic stem cells. BMC Dev. Biol. 8, 90.

Mammadov, B., Mammadov, R., Guler, M.O., Tekinay, A.B., 2012a. Cooperative effect of heparan sulfate and laminin mimetic peptide nanofibers on the promotion of neurite outgrowth. Acta Biomater. 8, 2077-2086.

Mammadov, R., Mammadov, B., Guler, M.O., Tekinay, A.B., 2012b. Growth factor binding on heparin mimetic peptide nanofibers. Biomacromolecules 13, 3311-3319.

Mammadov, B., Guler, M.O., Guler, M.O., Tekinay, A.B., 2013. Neural differentiation on synthetic scaffold materials. Biomater. Sci. 1, 1119-1137.

Martino, M.M., Tortelli, F., Mochizuki, M., Traub, S., Ben-David, D., Kuhn, G.A., Müller, R., Livne, E., Eming, S.A., Hubbell, J.A., 2011. Engineering the growth factor microenvironment with fibronectin domains to promote wound and bone tissue healing. Sci. Transl. Med. 3 (100), 100ra89.

Maude, S., Ingham, E., Aggeli, A., 2013. Biomimetic self-assembling peptides as scaffolds for soft tissue engineering. Nanomedicine (Lond.) 8, 823-847.

Mosesson, M.W., 2005. Fibrinogen and fibrin structure and functions. J. Thromb. Haemost. 3, 1894-1904.

Nomura, H., Baladie, B., Katayama, Y., Morshead, C.M., Shoichet, M.S., Tator, C.H., 2008. Delayed implantation of intramedullary chitosan channels containing nerve grafts promotes extensive axonal regeneration after spinal cord injury. Neurosurgery 63, 127-141. 
Novikova, L.N., Mosahebi, A., Wiberg, M., Terenghi, G., Kellerth, J.O., Novikov, L.N., 2006. Alginate hydrogel and matrigel as potential cell carriers for neurotransplantation. J. Biomed. Mater. Res. A 77, 242-252.

Orive, G., Tam, S.K., Pedraz, J.L., Halle, J.P., 2006. Biocompatibility of alginate-poly-L-lysine microcapsules for cell therapy. Biomaterials 27, 3691-3700.

Owen, S.C., Shoichet, M.S., 2010. Design of three-dimensional biomimetic scaffolds. J. Biomed. Mater. Res. A 94, 1321-1331.

Phelps, E.A., Landazuri, N., Thule, P.M., Taylor, W.R., Garcia, A.J., 2010. Bioartificial matrices for therapeutic vascularization. Proc. Natl. Acad. Sci. U. S. A. 107, 3323-3328.

Phillips, J.B., King, V.R., Ward, Z., Porter, R.A., Priestley, J.V., Brown, R.A., 2004. Fluid shear in viscous fibronectin gels allows aggregation of fibrous materials for CNS tissue engineering. Biomaterials 25, 2769-2779.

Plant, G.W., Woerly, S., Harvey, A.R., 1997. Hydrogels containing peptide or aminosugar sequences implanted into the rat brain: influence on cellular migration and axonal growth. Exp. Neurol. 143, 287-299.

Prang, P., Muller, R., Eljaouhari, A., Heckmann, K., Kunz, W., Weber, T., Faber, C., Vroemen, M., Bogdahn, U., Weidner, N., 2006. The promotion of oriented axonal regrowth in the injured spinal cord by alginate-based anisotropic capillary hydrogels. Biomaterials 27, 3560-3569.

Preston, M., Sherman, L.S., 2011. Neural stem cell niches: roles for the hyaluronan-based extracellular matrix. Front. Biosci. (Schol. Ed.) 3, 1165-1179.

Prestwich, G.D., 2008. Engineering a clinically-useful matrix for cell therapy. Organogenesis 4, 42-47.

Qiao, M., Chen, D., Ma, X., Liu, Y., 2005. Injectable biodegradable temperature-responsive PLGA-PEG-PLGA copolymers: synthesis and effect of copolymer composition on the drug release from the copolymer-based hydrogels. Int. J. Pharm. 294, 103-112.

Raeber, G.P., Lutolf, M.P., Hubbell, J.A., 2005. Molecularly engineered PEG hydrogels: a novel model system for proteolytically mediated cell migration. Biophys. J. 89, 1374-1388.

Rodriguez, F.J., Verdu, E., Ceballos, D., Navarro, X., 2000. Nerve guides seeded with autologous schwann cells improve nerve regeneration. Exp. Neurol. 161, 571-584.

Romano, N.H., Sengupta, D., Chung, C., Heilshorn, S.C., 2011. Protein-engineered biomaterials: nanoscale mimics of the extracellular matrix. Biochim. Biophys. Acta 1810, 339-349.

Rooney, G.E., Knight, A.M., Madigan, N.N., Gross, L., Chen, B., Giraldo, C.V., Seo, S., Nesbitt, J.J., Dadsetan, M., Yaszemski, M.J., Windebank, A.J., 2011. Sustained delivery of dibutyryl cyclic adenosine monophosphate to the transected spinal cord via oligo [(polyethylene glycol) fumarate] hydrogels. Tissue Eng. Part A 17, 1287-1302.

Sadr, N., Pippenger, B.E., Scherberich, A., Wendt, D., Mantero, S., Martin, I., Papadimitropoulos, A., 2012. Enhancing the biological performance of synthetic polymeric materials by decoration with engineered, decellularized extracellular matrix. Biomaterials 33, 5085-5093.

Sakiyama-Elbert, S., Johnson, P.J., Hodgetts, S.I., Plant, G.W., Harvey, A.R., 2012. Scaffolds to promote spinal cord regeneration. Handb. Clin. Neurol. 109, 575-594.

Silva, G.A., 2005. Nanotechnology approaches for the regeneration and neuroprotection of the central nervous system. Surg. Neurol. 63, 301-306.

Silva, G.A., Czeisler, C., Niece, K.L., Beniash, E., Harrington, D.A., Kessler, J.A., Stupp, S.I., 2004. Selective differentiation of neural progenitor cells by high-epitope density nanofibers. Science 303, 1352-1355. 
Silva, N.A., Salgado, A.J., Sousa, R.A., Oliveira, J.T., Pedro, A.J., Leite-Almeida, H., Cerqueira, R., Almeida, A., Mastronardi, F., Mano, J.F., Neves, N.M., Sousa, N., Reis, R.L., 2010. Development and characterization of a novel hybrid tissue engineering-based scaffold for spinal cord injury repair. Tissue Eng. Part A 16, 45-54.

Someya, Y., Koda, M., Dezawa, M., Kadota, T., Hashimoto, M., Kamada, T., Nishio, Y., Kadota, R., Mannoji, C., Miyashita, T., Okawa, A., Yoshinaga, K., Yamazaki, M., 2008. Reduction of cystic cavity, promotion of axonal regeneration and sparing, and functional recovery with transplanted bone marrow stromal cell-derived Schwann cells after contusion injury to the adult rat spinal cord. J. Neurosurg. Spine 9, 600-610.

Stensaas, S.S., Stensaas, L.J., 1978. Histopathological evaluation of materials implanted in the cerebral cortex. Acta Neuropathol. 41, 145-155.

Stokols, S., Tuszynski, M.H., 2006. Freeze-dried agarose scaffolds with uniaxial channels stimulate and guide linear axonal growth following spinal cord injury. Biomaterials 27, 443-451.

Tashiro, H., Iwata, H., Warnock, G.L., Takagi, T., Machida, H., Ikada, Y., Tsuji, T., 1997. Characterization and transplantation of agarose microencapsulated canine islets of Langerhans. Ann. Transplant. 2, 33-39.

Taylor, S.J., Rosenzweig, E.S., McDonald III, J.W., Sakiyama-Elbert, S.E., 2006. Delivery of neurotrophin-3 from fibrin enhances neuronal fiber sprouting after spinal cord injury. J. Control. Release 113, 226-235.

Tian, W.M., Zhang, C.L., Hou, S.P., Yu, X., Cui, F.Z., Xu, Q.Y., Sheng, S.L., Cui, H., Li, H.D., 2005. Hyaluronic acid hydrogel as Nogo-66 receptor antibody delivery system for the repairing of injured rat brain: in vitro. J. Control. Release 102, 13-22.

Tian, B., Liu, J., Dvir, T., Jin, L., Tsui, J.H., Qing, Q., Suo, Z., Langer, R., Kohane, D.S., Lieber, C.M., 2012. Macroporous nanowire nanoelectronic scaffolds for synthetic tissues. Nat. Mater. 11, 986-994.

Tobias, C.A., Dhoot, N.O., Wheatley, M.A., Tessler, A., Murray, M., Fischer, I., 2001. Grafting of encapsulated BDNF-producing fibroblasts into the injured spinal cord without immune suppression in adult rats. J. Neurotrauma 18, 287-301.

Tobias, C.A., Han, S.S., Shumsky, J.S., Kim, D., Tumolo, M., Dhoot, N.O., Wheatley, M.A., Fischer, I., Tessler, A., Murray, M., 2005. Alginate encapsulated BDNF-producing fibroblast grafts permit recovery of function after spinal cord injury in the absence of immune suppression. J. Neurotrauma 22, 138-156.

Tong, X., Yang, F., 2014. Engineering interpenetrating network hydrogels as biomimetic cell niche with independently tunable biochemical and mechanical properties. Biomaterials 35, 1807-1815.

Tysseling, V.M., Sahni, V., Pashuck, E.T., Birch, D., Hebert, A., Czeisler, C., Stupp, S.I., Kessler, J.A., 2010. Self-assembling peptide amphiphile promotes plasticity of serotonergic fibers following spinal cord injury. J. Neurosci. Res. 88, 3161-3170.

Tysseling-Mattiace, V.M., Sahni, V., Niece, K.L., Birch, D., Czeisler, C., Fehlings, M.G., Stupp, S.I., Kessler, J.A., 2008. Self-assembling nanofibers inhibit glial scar formation and promote axon elongation after spinal cord injury. J. Neurosci. 28, 3814-3823.

Ulrich, T.A., Juan Pardo, E.M., Kumar, S., 2009. The mechanical rigidity of the extracellular matrix regulates the structure, motility, and proliferation of glioma cells. Cancer Res. 69, 4167-4174.

Wang, T.W., Spector, M., 2009. Development of hyaluronic acid-based scaffolds for brain tissue engineering. Acta Biomater. 5, 2371-2384. 
Wang, X., He, J., Wang, Y., Cui, F.Z., 2012. Hyaluronic acid-based scaffold for central neural tissue engineering. Interface Focus. 2, 278-291.

Woerly, S., 2000. Restorative surgery of the central nervous system by means of tissue engineering using NeuroGel implants. Neurosurg. Rev. 23, 59-77.

Woerly, S., Petrov, P., Sykova, E., Roitbak, T., Simonova, Z., Harvey, A.R., 1999. Neural tissue formation within porous hydrogels implanted in brain and spinal cord lesions: ultrastructural, immunohistochemical, and diffusion studies. Tissue Eng. 5, 467-488.

Woerly, S., Pinet, E., de Robertis, L., Van Diep, D., Bousmina, M., 2001. Spinal cord repair with PHPMA hydrogel containing RGD peptides (NeuroGel). Biomaterials 22, 1095-1111.

Wong, D.Y., Leveque, J.C., Brumblay, H., Krebsbach, P.H., Hollister, S.J., Lamarca, F., 2008. Macro-architectures in spinal cord scaffold implants influence regeneration. J. Neurotrauma $25,1027-1037$.

Xu, X.M., Guenard, V., Kleitman, N., Bunge, M.B., 1995. Axonal regeneration into Schwann cell-seeded guidance channels grafted into transected adult rat spinal cord. J. Comp. Neurol. 351, 145-160.

Xu, X.M., Chen, A., Guenard, V., Kleitman, N., Bunge, M.B., 1997. Bridging Schwann cell transplants promote axonal regeneration from both the rostral and caudal stumps of transected adult rat spinal cord. J. Neurocytol. 26, 1-16.

Yu, L.M., Kazazian, K., Shoichet, M.S., 2007. Peptide surface modification of methacrylamide chitosan for neural tissue engineering applications. J. Biomed. Mater. Res. A $82,243-255$. 